IRSTI 29.27.07

https://doi.org/10.26577/phst-2019-2-p5

\title{
Plasma anisotropy around non-spherical conductive dust particles
}

\author{
G. Sukhinin*, M. Salnikov and A. Fedoseev \\ ${ }^{1}$ Institute of Thermophysics SB RAS, 1, Lavrentyev Ave., 630090, Novosibirsk, Russia \\ *e-mail: sukhinin@itp.nsc.ru
}

\begin{abstract}
This paper presents a computational model that allows self-consistent simulation of plasma distributions around isolated strongly charged dust grains with different geometries: spherical, ellipsoidal, and diskshaped dust particles. All particles in this work were considered conductive and were oriented so that cylindrical symmetry was preserved in the computational area. Dust particles of various shapes were placed in an external field, and the process of ion focusing and the formation of a wake behind them were studied. As a result of the calculation by this model, self-consistent distributions of the space charge and plasma potential around non-spherical dust particles, as well as the dependence of the main characteristics of the wake - the magnitude of the first potential maximum and its position - on the magnitude of the external field was obtained. The analysis of spatial distributions showed that near the dust grains of the same electric capacity almost identical spatial distributions of the space charge and potential are formed. When normalizing the dependence of the wake maximum value on the root of the electric capacitance, all data are described by a single parametric curve with good accuracy, which allows one to predict the plasma parameters around the dust particle without resorting to direct calculations.
\end{abstract}

Key words: dusty plasma, non-spherical dust particle, wake, plasma polarization, rod-like dust particles.

PACS numbers: 52.20.-j, 52.27.Lw.

\section{Introduction}

For several decades, the study of micron-sized solid particles placed in plasma has been conducted. Dusty plasma is studied in laboratory conditions [14], on the Earth [5], and in microgravity conditions at the International Space Station and in parabolic flights [6,7]. Numerical modeling also kept pace with the experimental study of dust particles - to calculate the plasma parameters around isolated dust particles, as well as dust particle clusters, a wide range of computational methods were developed and (or) applied: molecular dynamics [8], linear response [9,10], Monte Carlo, "Particle-In-Ccell" [11-13], etc.

Experimental and numerical methods have been studied many phenomena associated with dusty plasma: dust grains charging, the orientation of nonspherical dust particles in a discharge, selforganization of dust particles in a plasma, the formation of crystalline and liquid structures, phase transitions between these structures, wave effects in dust crystals and plane-transverse sound waves and many others. The study of dusty plasma easily obtained in the laboratory has opened a wide path for the experimental modeling of processes occurring in a gas, liquid, and solid matter at a kinetic level.

Based on the foregoing, it can be concluded that one of the main, fundamental phenomena in this complex medium is the self-organization of dust particles. Because of this, the most interesting are the reasons for the formation of ordered structures. Due to the fact that, using experimental studies, no such reasons were found, the already mentioned numerical methods were utilized to modulate the effect that a dust particle has on its surrounding.

Today, it is generally accepted that pseudoperiodic oscillating structures in potential and ion density are responsible for the dust particles ordering, which are formed behind a dust particle in a plasma stream or in an external electric field [1416]. This structure is called a wake, and is a consequence of ion focusing behind the dust grain. The most popular approaches for studying wakes are the already mentioned PIC [11-13] and LR 
$[9,10]$ numerical methods. However, a large number of papers are devoted to studying the distribution of the plasma potential near spherical dust particles, while in nature dust grains are rarely of such a regular shape.

In $[17-20]$, the authors of this article demonstrated the results of calculations of a model in which the distributions of space charges and potentials were determined in a self-consistent manner also around spherical particles. This article presents the dependences of the density distribution and the space charge potential, as well as the dependence of the dipole moment of the "dust particle - ion cloud" system for nonspherical particles: a flattened and elongated ellipsoid.

\section{Model}

Due to the fact that in the most detailed way the model has already been described in the works [1720], only its main points will be described later. For convenience, a particle whose geometry is an elongated ellipsoid will be called ellipsoidal, and the geometry of which is a flattened ellipsoid - diskshaped.

The geometry of the main computational domain was selected as a cube. An impenetrable sphere of radius $r_{0}<<\lambda_{i}$ was placed in the center of this cube - a figure simulating the dust particle itself. Here, $\lambda_{i}$ is the Debye ionic length. At the very beginning of the calculations, one ion was generated, the coordinates and velocities of which were set randomly. The speeds were set so that their values obeyed the Maxwell distribution.

To calculate the trajectory of this generated ion, Newton's motion equations were used. From these equations, the trajectory of this ion in a field induced by a dust particle and an external electrostatic field was calculated. During this movement, this ion could:

1) fall on a dust particle

2) fly beyond the computational domain

3) collide with a neutral atom.

The modeling region selected in the form of a cube was divided into cells $(i, j)$ according to the coordinates $\rho$ and $z$ respectively, so that the volume of each cell obeyed the following law:

$$
V_{i, j}=2 \pi \rho_{i} \Delta \rho_{i} \Delta z_{j} .
$$

The time $T_{i, j}$, that the observed ion spent in the space segment $(i, j)$, is fixed, normalized to the volume of the segment $V_{i, j}$, where the ion was located, after which obtained value is summed up with the already accumulated time statistics for this space element:

$$
n_{i}(i, j)=n_{i}(i, j)+\frac{T_{i, j}}{V_{i, j}} .
$$

The parameters in the system were calculated using dimensionless variables in which the dimensionless charge of the dust particle $\widetilde{Q}$ and the external electrostatic field $\widetilde{E}$ are given by the expressions:

$$
\widetilde{Q}=\frac{e^{2} Z_{d}}{\lambda_{i} k T_{i}}, \quad \widetilde{E}=\frac{e E \lambda_{i}}{k T_{i}}
$$

At the beginning of the calculation, a potential is set in the system as follows:

For a spherical particle:

$$
U_{0}(\rho, z)=-\frac{\widetilde{Q}}{r} \exp (-r)-\widetilde{E} z, r=\sqrt{\rho^{2}+z^{2}} .
$$

For an ellipsoidal particle:

$$
\begin{gathered}
U_{0, e l}(\rho, z)=-\frac{\tilde{Q}}{\sqrt{c^{2}-a^{2}}} \times \\
\mp \operatorname{Arth} \sqrt{\frac{\mathrm{c}^{2}-a^{2}}{\xi+c^{2}}} \exp (-r)-\tilde{E} z .
\end{gathered}
$$

For a disk-shaped particle:

$$
\begin{gathered}
U_{0, d i s k}(\rho, z)=-\frac{\tilde{Q}}{\sqrt{a^{2}-c^{2}}} \times \\
\times \operatorname{arctg} \sqrt{\frac{\mathrm{a}^{2}-c^{2}}{\xi+c^{2}}} \exp (-r)-\tilde{E} z .
\end{gathered}
$$

In this model, the figures are selected as ellipsoids of revolution, the two semi-axes of which are equal to each other. The length of the equal semi-axes is denoted as $a$, while the length of the third axis is denoted as $c$. In space, the dust particles were oriented so that the semi-axis $c$ was parallel to 
the external field vector. This orientation of the grain allows to maintain cylindrical symmetry in the model.

From the statistics accumulated over cylindrical segments, the dimensionless distribution of space charge $n(\rho, z)$, is calculated, which can be written in the form:

$$
n(\rho, z)=\frac{n_{i}(\rho, z)-n_{e}(\rho, z)}{n_{\infty}}
$$

From which the self-consistent potential in the system is calculated:

For a spherical particle:

$$
U(\rho, z)=-\frac{\widetilde{Q}}{r}+\iiint_{V_{\text {syst }}} \frac{n\left(\rho^{\prime}, \varphi^{\prime}, z^{\prime}\right) \rho^{\prime} d \rho^{\prime} d \varphi^{\prime} d z^{\prime}}{\left|\vec{r}-\vec{r}^{\prime}\right|}
$$

For an ellipsoidal particle:

$$
\begin{gathered}
U_{e l}(\rho, z)=-\frac{\tilde{Q}}{\sqrt{c^{2}-a^{2}}} \operatorname{Arth} \sqrt{\frac{c^{2}-a^{2}}{\xi+c^{2}}}+ \\
+\iiint_{V_{s s s t}} \frac{n\left(\rho^{\prime}, \phi^{\prime}, z^{\prime}\right) \rho^{\prime} d \rho^{\prime} d \phi^{\prime} d z^{\prime}}{\left|\vec{r}-\vec{r}^{\prime}\right|}-\tilde{E} z .
\end{gathered}
$$

For a disk-shaped particle:

$$
\begin{gathered}
U_{d i s k}(\rho, z)=-\frac{\tilde{Q}}{\sqrt{a^{2}-c^{2}}} \operatorname{arctg} \sqrt{\frac{\mathrm{a}^{2}-c^{2}}{\xi+c^{2}}}+ \\
+\iiint_{V_{s s s t}} \frac{n\left(\rho^{\prime}, \phi^{\prime}, z^{\prime}\right) \rho^{\prime} d \rho^{\prime} d \phi^{\prime} d z^{\prime}}{\left|\vec{r}-\vec{r}^{\prime}\right|}-\tilde{E} z .
\end{gathered}
$$

The general iterative scheme for finding a selfconsistent potential is as follows:

1) The ion trajectories in the potentials are calculated one by one (4-6), and how much time each ion spent in each element of space is taken into account. There is an accumulation of statistics.

2) By the formula (7), the distribution of the space charge is determined and by the formulas (810) the self-consistent potential of the system is calculated, and the charge of the dust particle is adjusted from the condition that the flows of ions and electrons are equal to its surface.

3) The calculation process goes into cycles, going to step one.

The algorithm was repeated until all spatial distributions, as well as the charge of the dust particle, reached the stationary regime, that is, at the moment when any further change could be characterized as a fluctuation.

\section{Results}

Figure 1 compares the spatial distributions of the space charge density $n(z, \rho)$ for particles of two types: a spherical dust particle of radius $r_{0}=2 \mu \mathrm{m}$ and an ellipsoidal dust particle of the aspect ratio $a$ $=1 \mu m c=5 \mu m$. Comparison is made for particles of precisely this size due to the fact that the conductive particles of this shape have the same electric capacity, which for particles of different types is determined by the formulas

$$
\begin{aligned}
& C_{e l}=\frac{\sqrt{c^{2}-a^{2}}}{\operatorname{Arch}(c / a)}-\text { ellipoidal; } \\
& C_{d}=\frac{\sqrt{a^{2}-c^{2}}}{\arccos (c / a)}-\text { disk-like; } \\
& C_{s}=r_{0} \text {-sphere. }
\end{aligned}
$$



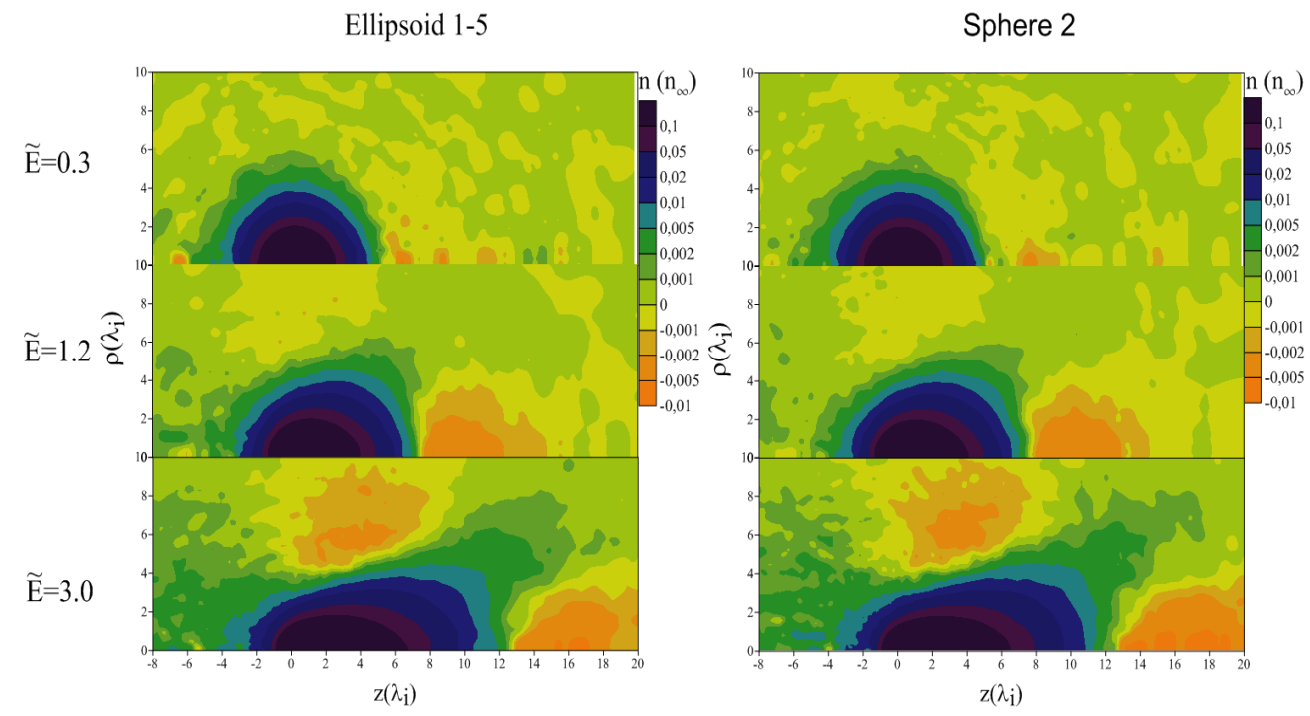

Figure 1 - The spatial distribution of the space charge density $n(z, \rho)$ for a spherical particle of radius $r_{0}=2 \mu \mathrm{m}$ and for an ellipsoidal particle of the aspect ratio $a=1 \mu \mathrm{mc}=5 \mu \mathrm{m}$ or various values of the external electrostatic field.
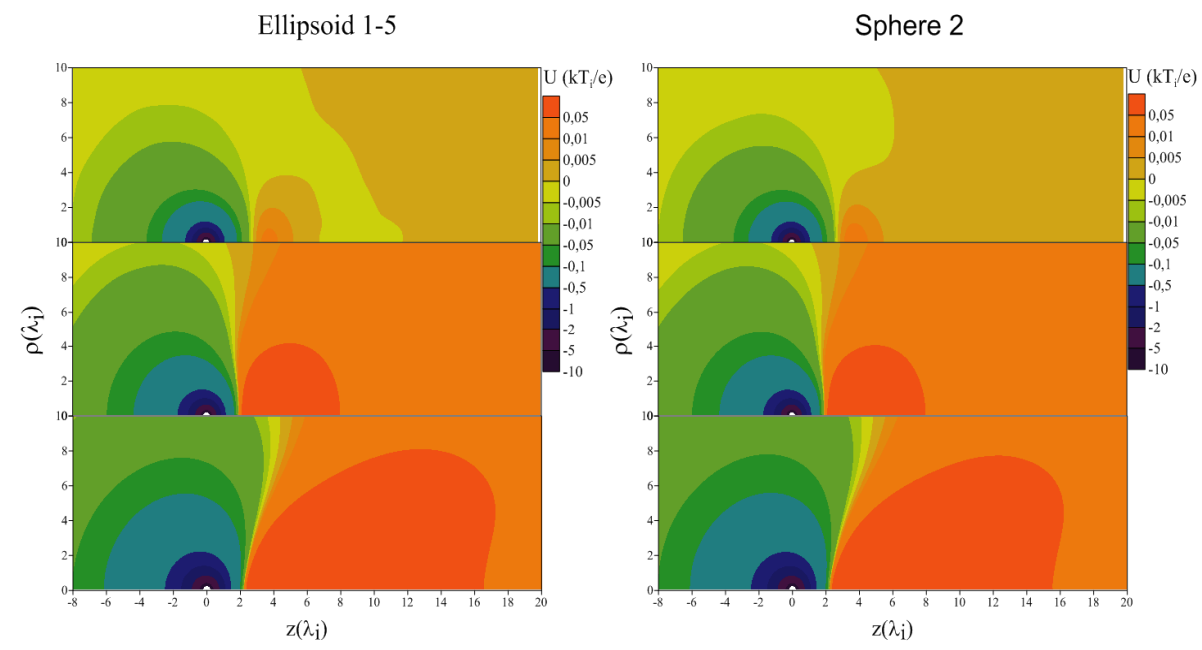

Figure 2 - The spatial distribution of electric potential $U(z, \rho)$ for a spherical particle of radius $r_{0}=2 \mu \mathrm{m}$ and for an ellipsoidal particle of the aspect ratio $a=1 \mu \mathrm{m} \mathrm{c}=5 \mu \mathrm{m}$ or various values of the external electrostatic field.

A comparison of the spatial distribution of the space charge density $n(z, \rho)$ shows that the ion focusing behind the dust particle occurs in exactly the same way for particles of different types. Neither the ion cloud itself, nor the disturbances in the plasma far from the dust grain have significant differences. A similar can be observed in Figure 2, where for the same field conditions a comparison of the spatial distributions of the electric potential $U(z, \rho)$ is presented. 

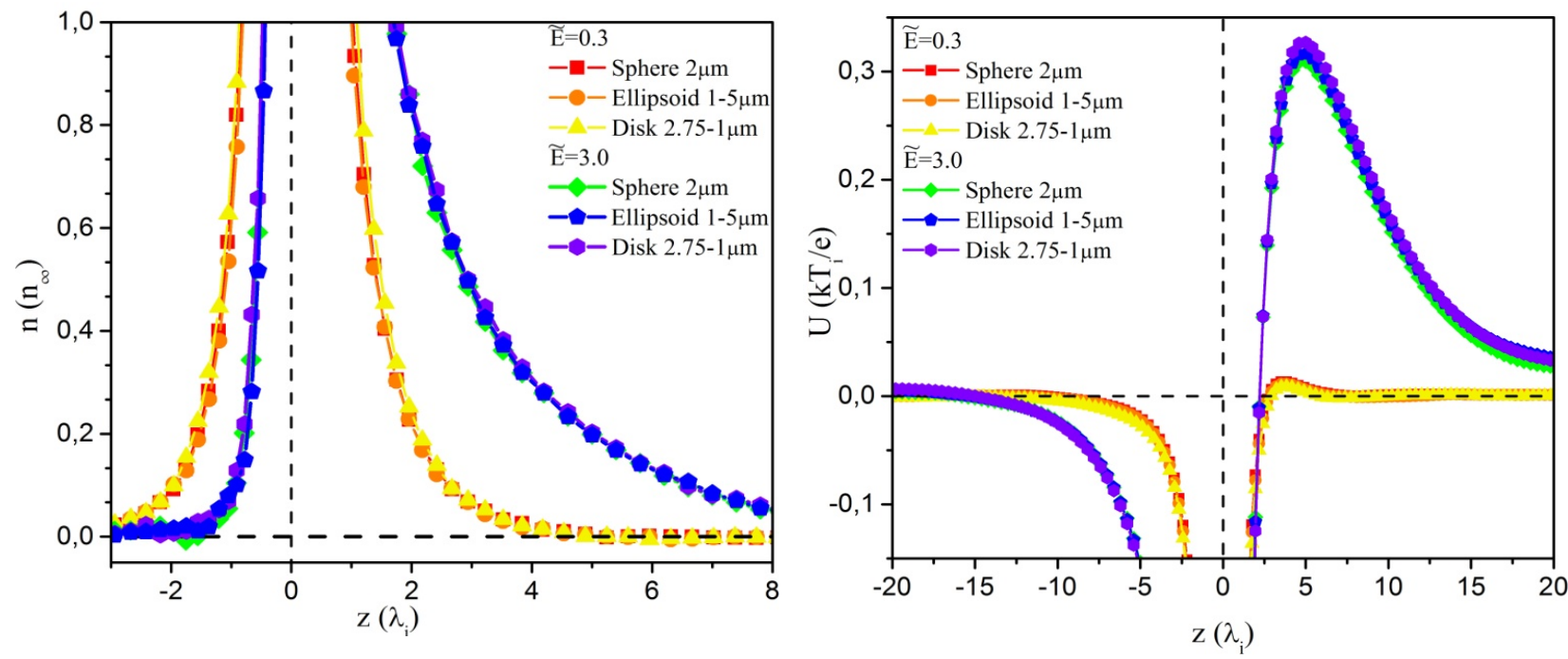

Figure 3 - The section of the spatial distribution of the space charge density $n(z, \rho=0)$ (left figure) and the potential $U(z, \rho=0)$ (right figure) for a spherical particle of radius $r_{0}=2 \mu \mathrm{m}$, an ellipsoidal particle of the aspect ratio $a=1 \mu m c=5 \mu m$ and a disk-shaped particle

of the aspect ratio $a=2.75 \mu m c=1 \mu m$ for various values of the external electrostatic field.

Figure 3 shows sections of the spatial distribution of the space charge density $n(z, \rho=0)$ and the potential $U(z, \rho=0)$. This representation allows comparisons for three types of particles: a sphere of radius $r_{0}=2 \mu \mathrm{m}$, an ellipsoidal particle of the aspect ratio $a=1 \mu m c=$ $5 \mu \mathrm{m}$ and a disk-shaped particle of the aspect ratio $a=$

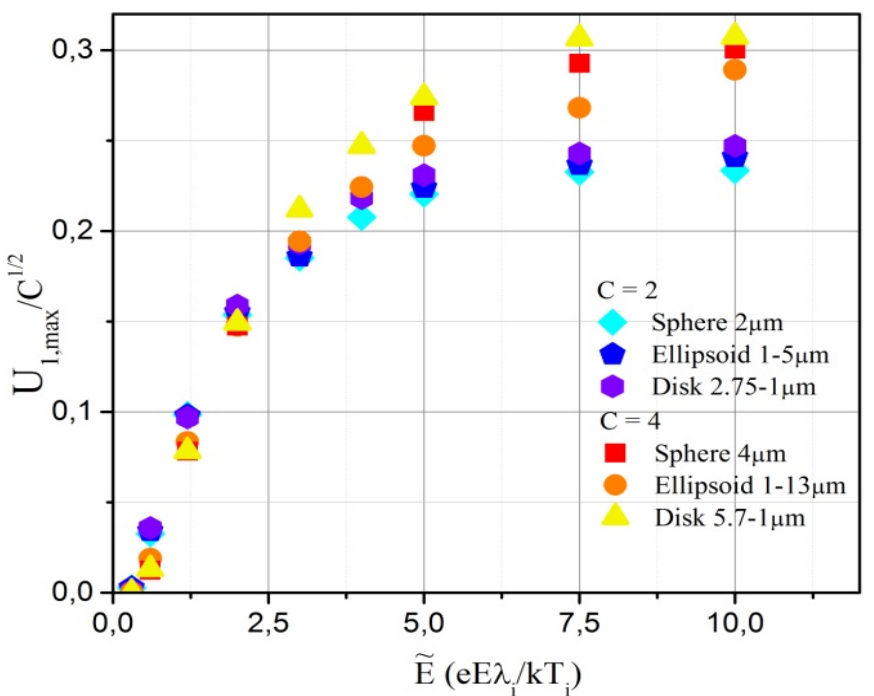

$2.75 \mu m c=1 \mu m$. All these conducting particles, when calculated by formulas (11), have the same capacitance $C=2$. Extensive comparison of the sections of the distributions for particles of different geometries shows that in this case there are no significant differences in the distributions.

Figure 4 - The dependence of the local maximum in the potential $U_{1, \max }$ (left figure) and the position of the local maximum $R_{l, \max }$ (right figure) on the magnitude of the external electrostatic field for spherical particles of radii $r_{0}=2 \mu \mathrm{m}$ and $4 \mu \mathrm{m}$, ellipsoidal particles of aspect ratios $a=1 \mu \mathrm{mc}=5 \mu \mathrm{m}$ and $a=1 \mu m c=13 \mu m$, as well as disk-shaped particles of aspect ratios $a=2.75 \mu m c=1 \mu m$ and $a=5.7 \mu m c=1 \mu m$. 
The data obtained in Figures 2 and 3 can be systematized, thus obtaining the dependence of the main characteristics of the wake (its maximum value and the location of this maximum) on the external field. Such dependences for particles of three grades are presented in Figure 4. Here, the comparison was expanded and dust particles with capacities equal to $C=4$, that is, spheres of radius $r_{0}=4 \mu \mathrm{m}$, an ellipsoidal particle of aspect ratio $a=1 \mu \mathrm{m} c=13$ $\mu m$ and a disk-shaped particle of the aspect ratio a $a$ $=5.7 \mu m c=1 \mu m$ were included in the comparison. The maximum of the wake in this case is normalized to the root of the electric capacity $\sqrt{C}$, which allows us to exclude the dependence of the wake characteristics on the dust particle charge. A similar normalization for spherical particles was considered in [19].

From this graph it can be established that there are no significant differences in the dependences of the wake characteristics on the external field for particles of different types. Thus, the approximation previously presented in [19] can be expanded:

$$
U_{1, \max }=A_{U} \widetilde{E} \sqrt{C l_{i}}\left(1+\left(\widetilde{E} / B_{U}\right)^{2}\right)^{-\gamma} \text {. }
$$

Where $A_{U}=0.028 \pm 0.0056, B_{U}=0.35 \pm 0.02$, $\gamma=0,75$. This approximation with good accuracy allows predicting the characteristics of the wake without resorting to calculations.

\section{Conclusions}

The existing computational model, which was previously demonstrated in several works, was expanded by a new computational unit capable of calculating the distribution of plasma parameters (spatial distributions of space charge and potential) around dust particles of non-spherical shape: elongated and oblate ellipsoids of revolution. In this model, the figures in the computational domain are oriented so that cylindrical symmetry is preserved in the computational area.

A new block of the computational model carried out a numerical study of the wake formation behind dust particles of different geometries and examined the main characteristics of this wake - the magnitude of its first positive maximum and its location relatively to the dust particle.

The calculation of the spatial distributions of the space charge and the plasma potential near the dust particle for conducting spherical, ellipsoidal, and disk-shaped dust grains of the same electric capacity at different values of the external electrostatic field showed that when dust particles are of the same electric capacity but are of different geometry are placed in a low-temperature plasma, the formation of a wake and ion focusing for such particles are identical. A more detailed examination of these spatial distributions (sections passing through the dust particle) also did not show significant differences.

As a result of multiple calculations, general dependences of the wake characteristics on the magnitude of the external electrostatic field for dust particles of various shapes were constructed. When normalizing the dependence of the wake maximum on the root of the electric capacity, all data are described with good accuracy by a single parametric curve, which allows one to predict the plasma parameters around the dust particle without resorting to direct calculations.

\section{References}

1. Hutchinson, I. H. (2002) Plasma Phys. Control. Fusion, 44, 9, 1953.

2. Melzer, A., Schweigert, V.A., Schweigert, I.V., Homann, A., Peters, S., and Piel, A. (1996) Structure and stability of the plasma crystal. Phys. Rev. E, 54, 1, R46-R49.

3. Melzer, A., Trottenberg, T., and Piel, A. (1994) Experimental determination of the charge on dust particles forming Coulomb lattices. Phys. Lett. A, 191, 34, 301-308.

4. Winske, D. (2001) Nonlinear wake potential in a dusty plasma. IEEE Trans. Plasma Sci., 29, 2, 191-197.

5. Khrapak, S.and Morfill, G. (2009) Basic Processes in Complex (Dusty) Plasmas: Charging, Interactions, and Ion Drag Force. Contrib. Plasma Phys., 49, 148-168.

6. Verheest, F. (2000) Waves in Dusty Space Plasmas. Kluwer Academic, Dordrecht.

7. Zhukhovitskii, D.I., Fortov, V.E., et al (2012) Nonviscous motion of a slow particle in a dust crystal under microgravity conditions. Physical Review E 86, 1, 016401.

8. Vladimirov, S.V., Maiorov, S.A., Cramer, N.F. (2003) Kinetics of plasma flowing around two stationary dust grains. Phys. Rev. E., 67, 016407. 
9. Dewar, R.L. and Leykam, D. (2012) Dressed test particles, oscillation centres and pseudo-orbits. Plasma Phys. Control. Fusion, 54, 014002.

10. Kompaneets, R., Morfill, G.E., and Ivlev, A.V. (2016) Interparticle Attraction in 2D Complex Plasmas. Phys. Rev. Lett., 116, 125001.

11. Ludwig, P., Miloch, W.J., Kahlert, H., Bonitz, M. (2012). On the wake structure in streaming complex plasmas. New J. Phys., 14, 053016.

12. Hutchinson, I.H. (2011) Nonlinear collisionles plasma wakes of small particles. Phys. Plasmas, ,18, 032111.

13. Hutchinson, I.H. (2012) Intergrain forces in low-Mach-number plasma wakes. Phys. Rev. E, 85, 066409.

14. Lampe, M., Joyce, G., Ganguli, G., and Gavrishchaka, V. (2000) Interactions between dust grains in a dusty plasma. Phys. Plasmas, 7, 10, 3851-3861.

15. Thomas, H., Morfill, G.E., Demmel, V., Goree, J., Feuerbacher, B., Mohlmann, D. (1994) Plasma Crystal: Coulomb Crystallization in a Dusty Plasma. Phys. Rev. Lett., 73, 652.

16. Chu, J.H. and Lin, I. (1994) Direct observation of Coulomb crystals and liquids in strongly coupled rf dusty plasmas. Phys. Rev. Lett, 72, 4009.

17. Sukhinin, G. I., Fedoseev, A. V., Salnikov, M. V., et al. (2017) Plasma anisotropy around a dust particle placed in an external electric field. Phys. Rev. E., 95, 063207.

18. Sukhinin, G.I., Fedoseev, A.V., Salnikov, M.V. (2016) Polarization of a Dust Particle and Surrounded Plasma in an External Electric Field. Contrib. Plasma Phys, 56, 5, 397-402.

19. Sukhinin, G.I., Fedoseev, A.V., Salnikov, M.V. (2019) The influence of dust particle geometry on its charge and plasma potential. Contributions to Plasma Physics, 201800153.

20. Sukhinin, G.I., Fedoseev, A.V., Salnikov, M.V. (2019) Effect of ion mean free path length on plasma polarization behind a dust particle in an external electric field. Contributions to Plasma Physics, 201800152. 